\title{
Ibrutinib Is Effective in the Treatment of Autoimmune Haemolytic Anaemia in Mantle Cell Lymphoma
}

\author{
Aliénor Galinier Vincent Delwail Mathieu Puyade \\ CHU de Poitiers, Service d'Onco-Hématologie et Thérapie Cellulaire, Poitiers, France
}

\section{Keywords}

Mantle cell lymphoma A Autoimmune hemolytic anaemia $\cdot$ Ibrutinib

\begin{abstract}
Autoimmune haemolytic anaemia (AIHA) in mantle cell lymphoma (MCL) is a rare but lifethreatening complication. To date, there are no relevant data for treatment of AIHA in MCL. Ibrutinib, which has been approved for relapse/refractory $\mathrm{MCL}$, is an immunomodulatory drug inhibiting Th2 activation and consequently the production of autoantibodies. We report a case of MCL with AIHA in which this form of anaemia was not controlled with the usual chemotherapy. Ibrutinib was used when MCL with AIHA relapsed, and it allowed rapid remission of AIHA and rapid discontinuation of steroid therapy.

(C) 2017 The Author(s) Published by S. Karger AG, Basel
\end{abstract}

\section{Introduction}

Mantle cell lymphoma (MCL) is a rare subtype of non-Hodgkin B-cell lymphoma [1]. Autoimmune haemolytic anaemia (AIHA) is a relatively uncommon disorder with an estimated incidence in adults of $0.8-3$ per $10^{5}$ /year, a prevalence of $17: 100,000$ and a mortality rate of $11 \%[2]$.

Recent reviews have focused on MCL treatment, without any data on haemolytic anaemia $[1,3]$. Cases of MCL with AIHA are usually excluded from clinical trials. In fact, this com- 


\section{Case Reports in Oncology}

plication is rare in MCL in contrast with other lymphoproliferative disorders such as chronic lymphocytic leukemia [4]. The treatment of AIHA in MCL is not well defined, but immune chemotherapy is usually proposed to control the tumor and also hemolysis. Ibrutinib, a Bruton's tyrosine kinase inhibitor, was recently approved for the therapy of relapse/refractory MCL. Activation of the B-cell antigen receptor (BCR) signaling pathway contributes to the initiation and maintenance of B-cell malignancies and autoimmune diseases. Ibrutinib has been shown to inhibit production of autoantibodies in murine models of autoimmunity [5]. Ibrutinib is also an immunomodulatory drug, irreversibly binding to ITK, thereby inhibiting Th2 activation. This inhibition is specific to Th2-polarized CD4 T cells, as resting lymphocyte kinase remains functional, thereby providing a compensatory platform for activation of Th1 and CD8 T cells. Recent data have demonstrated that CD4 T-cell populations isolated from chronic lymphocytic leukemia patients are skewed toward a Th1 profile after exposure to ibrutinib [6]. Recent studies have suggested that ibrutinib is an effective treatment of AHAI in chronic lymphocytic leukaemia $[7,8]$ and could also be effective for AHAI treatment related to MCL.

\section{Case Presentation}

A 75-year-old female was diagnosed with MCL with medullary involvement in August 2001. The karyotype showed characteristic translocation $(11 ; 14)$. No molecular test was performed. She was under medical supervision. In May 2002, given the evolution of her disease, she received 6 cycles of R-DHAP (rituximab, cisplatin, cytarabine, and dexamethasone) followed by autologous stem-cell transplantation. Complete remission was observed. In 2006, however, she presented a lymph node relapse associated with an AIHA. She received 6 cycles of R-HAD (rituximab, bortezomib, aracytin, and dexamethasone), which allowed partial remission without AIHA control. Between February 2007 and February 2009, she received maintenance treatment by bortezomib, rituximab, and cyclophosphamide and achieved complete remission of MCL and AIHA.

In July 2015, a complete blood count showed a WBC of $4 \times 10^{9} / \mathrm{L}$ with $80 \%$ abnormal lymphocytes, haemoglobin $79 \mathrm{~g} / \mathrm{l}$, and a platelet count of $162 \times 10^{9} / \mathrm{L}$. MCL relapse was confirmed by blood immunophenotype. AIHA was confirmed by an elevated lactate-dehydrogenase (LDH) level, reticulocyte count of $250 \times 10^{9} / \mathrm{L}$, elevated total and direct bilirubin, low serum haptogloblin level, and a direct antiglobulin test was positive with 3+ reactivity with anti-IgG. She was ECOG 0 and showed no tumoral syndrome except for a 2-centimeter splenomegaly under the costal edge. The patient was diagnosed with AIHA and relapse of MCL and received prednisone $1 \mathrm{mg} / \mathrm{kg}$. One week later, she had a mild improvement in haemoglobin to $92 \mathrm{~g} / \mathrm{l}$ and started ibrutinib treatment at $560 \mathrm{mg} /$ day.

One month later, a complete blood count demonstrated an improvement in anaemia (haemoglobin $106 \mathrm{~g} / \mathrm{L}$ ) and haemolysis (normal LDH and bilirubin levels) and disappearance of the splenomegaly. Steroid therapy was stopped. Three weeks later, a blood test confirmed the improvement in haemoglobin to $110 \mathrm{~g} / \mathrm{L}$ and haemolysis with normalization of LDH and total bilirubin levels.

Six months later, the patient had a normal level of haemoglobin at $120 \mathrm{~g} / \mathrm{L}$ but without hemolysis stigmata. However, a direct antiglobulin test remained positive. Ibrutinib was well tolerated with no adverse events reported.

AIHA is a rare but life-threatening complication in MCL. In this report, ibrutinib allowed rapid AIHA remission, whereas previous AIHA had required several months of steroid ther- 
apy. In this situation, rapid discontinuation of steroid therapy may be a safer approach to AIHA treatment of patients with MCL. To our knowledge, this is the first report of ibrutinib therapy aimed at controlling AIHA in MCL.

\section{Conclusion}

Ibrutinib is effective in the treatment of AHAI in MCL.

\section{Acknowledgment}

We would like to thank Jeffrey Arsham for revision of the English language manuscript and the APEMSA (Association Pictave pour l'Etude des Maladies du Sang) for its help.

\section{Statement of Ethics}

Written consent was signed by the patient.

\section{Disclosure Statement}

The authors declare that they have no conflicts of interest to disclose.

\section{Author Contribution}

A.G., V.D., and M.P. performed and designed the research. All authors wrote the manuscript.

\section{References}

Cheah CY, Seymour JF, Wang ML: Mantle cell lymphoma. J. Clin. Oncol 2016;34:1256-1269.

Zanella A, Barcellini W: Treatment of autoimmune hemolytic anemias. Haematologica 2014;99:15471554.

- 3 Campo E, Rule S: Mantle cell lymphoma: evolving management strategies. Blood 2015;125:48-55

- 4 Zent CS, Ding W, Reinalda MS, Schwager SM, Hoyer JD, Bowen DA, et al: Autoimmune cytopenia in chronic lymphocytic leukemia/small lymphocytic lymphoma: changes in clinical presentation and prognosis. Leuk Lymphoma 2009;50:1261-1268.

-5 Honigberg LA, Smith AM, Sirisawad M, Verner E, Loury D, Chang B, et al: The Bruton tyrosine kinase inhibitor PCI-32765 blocks B-cell activation and is efficacious in models of autoimmune disease and B-cell malignancy. Proc Natl Acad Sci USA 2010;107:13075-13080.

-6 Dubovsky JA, Beckwith KA, Natarajan G, Woyach JA, Jaglowski S, Zhong Y, et al: Ibrutinib is an irreversible molecular inhibitor of ITK driving a Th1-selective pressure in T lymphocytes. Blood 2013;122:2539-2549.

7 Manda S, Dunbar N, Marx-Wood CR, Danilov AV: Ibrutinib is an effective treatment of autoimmune haemolytic anaemia in chronic lymphocytic leukaemia. Br J Haematol 2015;170:734-736.

8 Molica S, Levato L, Mirabelli R: Chronic lymphocytic leukemia, autoimmune hemolytic anemia and ibrutinib: a case report and review of the literature. Leuk Lymphoma 2016;57:735-737. 